\title{
A Survey on Efficient Clustering Methods with Effective Pruning Techniques for Probabilistic Graphs
}

\author{
M.Balaganesh \\ Associate Professor, Dept of CSE \\ Sembodai Rukmani Varatharajan Engineering \\ College, \\ Sembodai, Tamilnadu
}

\author{
G.Bharathikannan \\ PG Scholar, Dept of CSE \\ Sembodai Rukmani Varatharajan Engineering \\ College, \\ Sembodai,Tamilnadu
}

\begin{abstract}
This paper provides a survey on K-NN queries, DCR query, agglomerative complete linkage clustering and Extension of edit-distance-based definition graph algorithm and solving decision problems under uncertainty. This existing system give an beginning to Graph agglomeration aims to divide information into clusters per their similarities, and variety of algorithms are planned for agglomeration graphs, the pKwik Cluster algorithm, spectral agglomeration, k-path agglomeration, etc. However, very little analysis has been performed to develop efficient agglomeration algorithms for probabilistic graphs. Finally, The Graph algorithm to understand how to mining can be done efficiently. This survey introduced to design algorithm for searching and to evaluate the algorithm throw analysis.
\end{abstract}

\section{General Terms}

Graph mining, PGM, PPI

\section{Keywords}

Cluster, Probabilistic Graphs, pKwik Cluster algorithm.

\section{INTRODUCTION}

\subsection{Data Mining}

Data mining is the process of analyzing data to extract information from the data. The data mining be able to help in predicting a trend or value, classifying, categorizing the data, and in finding correlations, patterns from the data set. Data can be mine irrespective of storage format in which it is stored. The data can be stored in flat files, spreadsheets, database tables, or some other storage format. The storage format is not important, but its applicability to the problem to be solved is more important. Data mining is basically one of the steps in the process of knowledge discovery in database (KDD).Knowledge discovery process is basically divided in 4 steps:

1) Selection-Identify the data.

2) Pre-Processing-Cleanse and profile the data.

3) Transformation-Required for data preparation and data mining.

4) Evaluation-Result of data mining.

\subsubsection{Techniques in Data Mining}

The following major data mining tasks,

- Association-Pattern discovery based on relationship of a particular item on the other item in the same transaction.

- Classification-Used to classify each item in a set of data into one predefined set of classes or groups.

- Clustering-Clustering means similar characteristics of objects are grouped.
- Prediction-Discovers relationship among independent variables and relationship between dependent and independent variables.

- Sequential Pattern-Discover similar pattern in database.

\subsubsection{Association Rule Mining}

Association rule mining is a type of data mining method. Association rule mining is done to extract interesting correlations, associations, patterns among items in the transaction database or other data repositories. For example an association rule fruit $\Rightarrow$ milk generated from the transaction database of a grocery store can help in formulating marketing strategy around the rule. Association rules are widely used in various areas such as telecommunication networks, marketing and risk management, and inventory control.

These data could be analyzed to learn the purchasing trend of the customer. Such valuable insight can be used to support variety of business-related applications such as marketing and promotion of the products, inventory management etc. Besides markets based data analysis, association rules can also be mined for the field of medical diagnosis, bioinformatics, web mining and scientific data analysis. All the above fields deal with the huge amount of input data, whose locations could be distributed. Processing such huge data requires lots of resources and time.

The algorithm generates an extremely large number of association rules in many cases and sometimes the association rules are very large. It becomes nearly impossible for the users to comprehend or validate such large number of complex association rules, thereby limiting the worth of the data mining results. Thus the concept of generating only "interesting" rules, generating only "non-redundant" rules, or generating only those rules which satisfies certain criteria are reached. The criteria could be confidence, coverage, leverage, lift or strength.

The parallel association rule mining can be categorized in two sections. The first is data parallelism in which the input data set could be divided among the participating node to generate the rules. The second method is of dividing the charge among the nodes so that each node will access the whole input data set for generating the rules.

\section{RELATED WORK}

Graph mining has gained considerable attention for a broad range of applications, such as social networks, protein-protein interaction networks, road networks, etc.

Uncertainty is inescapable in real-world applications: The existing system able to nearly never predict with certainty what's going to happen within the future and even within the gift and therefore the past, numerous necessary aspects of the planet are not determined with certainty. Applied mathematics offers United States the fundamental foundation to model our 
beliefs concerning the various potential states of the planet, and to update these beliefs as new proof is obtained. These beliefs are often combined with individual preferences to assist guide our actions, and even in choosing that clarification to create. Whereas applied mathematics has existed since the seventeenth century, our ability to use it.

Effectively on massive issues involving several inter-related variables is fairly recent, and is due mostly to the event of a framework called Probabilistic Graphical Models (PGMs)[1][2]. This framework, that spans strategies evocative of theorem networks and Mark off random fields, uses ideas from distinct information structures in computing to with efficiency write in code and manipulates likelihood distributions over high-dimensional areas, typically involving lots of or maybe several thousands of variables. These strategies are employed in a vast vary of application domains, that include: internet search[7], medical and fault designation, image considerate, reconstruction of biological networks, speech recognition, language process, decryption of messages sent over a loud line, mechanism routing, and lots of a lot of. The PGM framework provides a vital tool for anyone World Health Organization desires to find out a way to reason coherently from controlled and vociferous observations.

In a probabilistic graph, any two edges ei and ej are called conditionally Independent if $\mathrm{p}(\mathrm{ei}, \mathrm{ej})=\mathrm{p}(\mathrm{ei}) \mathrm{p}(\mathrm{ej})$, and conditionally dependent if $p(e i, e j) \neq p(e i) p(e j)$. For the regular probabilistic graph model, any two edges are conditionally independent of each other. Typically, beginners and mutual exclusion among adjacent edges [3][4] area unit generally determined in numerous graph headed applications. As one of the fundamental data processing techniques, bunch is wide employed in numerous graph analysis applications [5][6] admire community finding, index construction, etc. This paper focuses on clustering correlated probabilistic graphs that aims to partition the vertices into many disconnected clusters with high intra-cluster and low inter-cluster similarity, as illustrated. Next, The existing system are going to inspire the matter of bunch correlative probabilistic graphs exploitation many applications.

In Protein-Protein Interaction (PPI) networks [1], the interaction between 2 proteins is mostly established with a probability property thanks to the limitation of observation strategies. Additionally, it's been verified that the interaction between super molecules A and B can influence the interaction between protein and another protein C, if A, B and $\mathrm{C}$ have some regular options. It's been verified that the likelihood of pair wise interaction and correlation among edges will be derivative from applied mathematics models. Bunch applied to such related to probabilistic protein-protein interaction network knowledge is useful to find complexes to research the structure properties of the PPI Network.

To cluster a correlate probabilistic graph G, a possible world graph $\mathrm{Gi}$ of $\mathrm{G}$ can be sculptural as a settled internal representation sampled from the correlate probabilistic graph in step with the chance distribution. The edit distance $\mathrm{D}(\mathrm{Gi}$ ,Q) [6] from Gi to the cluster graph note is outlined because the variety of edges that require to be superimposed or removed to remodel $\mathrm{Gi}$ into $\mathrm{Q}$. By evaluating all the potential world graph instances, the expected edit distance, denoted as $\mathrm{D}(\mathrm{G}, \mathrm{Q})$ is obtained and viewed as a activity to gauge the difference from a correlate probabilistic graph to the cluster graph. Hence, a smaller difference implies a a lot of precise result, and our objective turns to the goal of decision a cluster graph $Q$ that may minimize $D(G, Q)$. However, it's very long if the existing system has a tendency to calculate the expected edit distance by considering all impending world graphs. To resolve this drawback, The existing system have a tendency to propose a completely unique estimation model which needs the high-octane generation of a position access order once hard conditional chances. The estimation model has obvious error bounds.

\subsection{PROBABILISTIC GRAPHS}

To the best of our knowledge, first to define and study the problem of clustering probabilistic graphs using the possibleworlds semantics. However, uncertain data management and graph mining has aggravated many studies in the data mining and database [3] community. Highlight some of this work here.

\subsubsection{Graph and Probabilistic-Graph Mining}

Clustering and partitioning of deterministic graphs has been an active area of research. For an extensive survey on the topic see and the references therein. Most of these algorithms can be used to handle probabilistic graphs, either by considering the edge probabilities as weights, or by setting a threshold value to the probabilities of the edges and ignoring any edge with probability below this threshold. The disadvantage of the first approach is that once probabilities are interpreted as weights[4], then no other weights can be taken into consideration (unless the probabilities are multiplied with edge weights - in which case this composite weight has no interpretation). The disadvantage of the second approach is that there is no principled way of deciding what the right value of the threshold is. Although both the above methodologies would result in an algorithm that would output some node clustering, this algorithm, contrary to ours, would not optimize an objective defined over all possible worlds of the input probabilistic graph. Further, various graph mining problems have been studied recently assuming uncertain graphs. For example, Hintsanen and Toivonen looked at the problem of finding the most reliable sub graph, and Zou et al. considered the problem of finding frequent sub graphs of an input probabilistic graph. More recently, Potamias et al. proposed new robust distance functions between nodes in probabilistic graphs[3] that extend shortest path distances from deterministic graphs and proposed methods to compute them efficiently[5]. The problem of finding shortest paths in probabilistic graphs based on transportation networks has also been considered. The intersection between the above methods and ours is that all of them deal with probabilistic graphs. However, the graph-clustering task under the possible-worlds semantics has not yet been addressed by researchers in probabilistic graph mining.

CR. Jin, L. Liu, B. Ding, and H. Wang, "Distance-constraint reachability computation in uncertain graphs,"[2] Querying and mining uncertain graphs has become a progressively more important research topic. In the most common uncertain graph model, edges are autonomous of one another, and each edge is associated with a probability that indicates the likelihood of its survival. This gives rise to using the possible world semantics to model uncertain graphs. A possible graph of an uncertain graph $G$ is a possible instance of $G$. A possible graph contains a subset of edges of $G$, and it has a weight which is the product of the probabilities of all the edges it has. For example, illustrates an uncertain graph $\mathrm{G}$, and three of its possible graphs G1, G2 and G3, each with a weight. A primary question for uncertain graphs is to classify and compute reachability between any two vertices. In a deterministic directed graph, the reachability query, which ask whether one vertex can reach another one, is the source for a variety of databases (XML/RDF) and network applications. For uncertain graphs, reachability is not a simple Yes/No 
question, but instead, a probabilistic one. exclusively, reachability from vertex $\mathrm{s}$ to vertex $\mathrm{t}$ is expressed as the overall probability of those possible graphs of $G$ in which $s$ can reach $t$. For uncertain graph $G$, we can see that $s$ can reach $t$ in its possible graphs $\mathrm{G} 1$ and $\mathrm{G} 2$ but not in G3; if we specify all the possible graphs of $\mathrm{G}$ and add up the weights of those possible graphs where $\mathrm{s}$ can reach $\mathrm{t}$, we get $\mathrm{s}$ can reach $\mathrm{t}$ with probability 0.5104 . The simple reachability in uncertain graphs has been widely deliberate in the context of network reliability and system engineering.

In this paper, we study a more generalized and informative distance-constraint reachability (DCR) query difficulty, that is: Given two vertices $s$ and $t$ in an uncertain graph $G$, what is the probability that the distance from $s$ to $t$ is less than or equal to a user-defined threshold d Basically, the distanceconstraint reachability (DCR) between two vertices requires them not only to be connected in the possible graphs, but also to be close enough. The threshold $\mathrm{d}$ is selected to be 2 , then, $\mathrm{t}$ is considered to be unreachable from 2 in G2 (under this distance constraint). obviously, DCR query enables a more informative categorization and interrogation of the reachability among any two vertices. At the same time, the simple reachability also becomes a special case of the distance-constraint reachability (considering the case where the threshold $d$ is larger than the length of the longest path, or simply the sum of all edge weights in G). Distance-constraint reachability plays a main and even critical role in a wide range of applications. In a variety of real-world emerging communication networks, DCR is essential for analyzing their reliability and communication quality. For example, in peer to-peer (P2P) networks, such as Free net and Gnutella, the communication between two nodes is only allowed if they are separated by a small number of intermediate hops (to avoid jamming). In such situation, as the uncertain graph naturally models the link crash probability, the DCR query serves as the basic tool to interrogate the probability whether one node can communicate with another, and to study the network reliability in general. Indeed, such diameter-constrained (or hop-constrained) reliability has been proposed in the context of communication network reliability though its computation remains difficult

The Horvitz-Thomson type estimator and effectively combines a deterministic recursive computational procedure with a sampling process to boost the estimation accuracy. These are the important concept of this paper.

\section{EXISTING SYSTEM}

Spectral clustering relies on the Eigen structure of a graph Laplacian matrix to partition vertices into disjoint clusters, with points in the matching cluster having high similarity and points in different clusters having low similarity. The rationality of the spectral clustering method was analyzed by Bach et al. They derived new cost functions for spectral clustering based on measures of error between a given partition and a solution of the spectral relaxation

The problem of Existing system is extremely time-consuming [8] if we calculate the expected edit distance by considering all possible world graphs and Ignoring relationship correlations will lead to incorrect results.

\section{CONCLUSION}

In this paper studied how to improve ranking of an instantfuzzy search system by considering proximity information when need to compute top-k[10] answers. We studied how to adapt existing solutions to solve this problem, including computing all answers, doing early termination, and indexing term pairs. The proposed technique is to index important phrases to avoid the large space overhead of indexing all word grams and presented an incremental-computation algorithm for finding the indexed phrases in a query efficiently [5][6], and studied how to compute and rank the segmentations consisting of the indexed phrases when compared with techniques to the instant fuzzy adaptations of basic approaches. Then conducted a very thorough analysis by considering space, time, and relevancy tradeoffs of these approaches.

\section{REFERENCES}

[1] Bonchi.F, Gionis.A, Kollios.G and Potamias.M, PVLDB, vol. 3, no. 1, pp. 997-1008, Sept. 2010. "K-nearest neighbors in uncertain graphs,"

[2] Ding.B, Jin.R, Liu.L and Wang.H, PVLDB, vol. 4, no. 9, pp. 551-562, Jun. 2011. "Distance-constraint reachability computation in uncertain graphs,"

[3] Chen.L, Wang.G, Wang.H and Yuan.Y, PVLDB, vol. 5, no. 9, pp. 800-811, May 2012. "Efficient subgraph similarity search on large probabilistic graph databases,"

[4] Hua.M and Pei.J, in Proc. 13th Int. EDBT, New York, NY, USA, 2010, pp. 347-358."Probabilistic path queries in road networks: Traffic uncertainty aware path selection,"

[5] Flynn.P.J, Jain.A.K and Murty.M.N, ACM Comput. Surv. vol. 31, no. 3, pp. 264-323,Sept. 1999 "Data clustering: A review,"

[6] Shamir.R, Sharan.R, and Tsur.D, Discrete Appl. Math., vol. 144, no. 1-2,pp. 173-182, 2004."Cluster graph modification problems,"

[7] Cetindil.I, Esmaelnezhad.J, Li.C, and Newman.D, in WebDB, 2012, pp. 7-12. "Analysis of instant search query logs,"

[8] [8]Miller.R.B, in Proceedings of the December 9-11, 1968, fall joint computer conference, part I, ser. AFIPS '68 (Fall, part I). New York, NY, USA: ACM, 1968, pp. 267-277. "Response time in man-computer conversational transactions,"

[9] Henzinger.M.R, Marais.H, Moricz.M and Silverstein.C, "Analysis of a very large web search engine query log,"

[10] Ackermann.M.R, Blömer.J, Kuntze.D, and Sohler.C, Algorithmica, vol. 69, no. 1, pp. 184-215, May 2014."Analysis of agglomerative clustering,"

[11] Broschart.A, Schenkel.R, Theobald.M, won Hwang.S and Weikum.G, in SPIRE, 2007, pp. 287-299. "Efficient text proximity search,"

[12] Shi.S, Suel.T, Wen.J.R, Yan.H and Zhang.F. in CIKM, 2010, pp. 1229- 1238."Efficient term proximity search with term-pair indexes,"

[13] Shi.S, Wen.J.R, Yu.N and Zhu.M. in CIKM, 2008, pp. 679-688. "Can phrase indexing help to process nonphrase queries?"

[14] Jain.A and Pennacchiotti.M, in COLING, 2010, pp. 510518. "Open entity extraction from web search query $\operatorname{logs}, "$

[15] Grabski.K and Scheffer.T, in SIGIR, 2004, pp. 433-439. "Sentence completion," 